\title{
A GIS-based Optimal Facility Location Framework for Fast Electric Vehicle Charging Stations
}

\author{
Usman Zafar \\ Qatar Environment and \\ Energy Research Institute, \\ Hamad Bin Khalifa University \\ PO Box: 34110 Doha - Qatar \\ uzafar@hbku.edu.qa
}

\author{
I. Safak Bayram \\ Dept. of Electronic and Electrical Engineering \\ University of Strathclyde \\ Glasgow, United Kingdom, G1 1XQ \\ safak.bayram@strath.ac.uk
}

\author{
Sertac Bayhan \\ Qatar Environment and \\ Energy Research Institute \\ Hamad Bin Khalifa University \\ PO Box: 34110 Doha - Qatar \\ sbayhan@hbku.edu.qa
}

\begin{abstract}
Deeper decarbonization of the transport sector requires building a wide coverage electric vehicle charging network that can meet driver's mobility patterns and refueling habits in a seamless manner. Currently, major market players mainly deploy chargers at existing public parking spaces at hotels, shopping centers, etc. On the other hand, gas/petroleum retail business is a century-old industry and "optimized" to serve the refueling needs of the drivers and they come to the forefront as "good" locations to site chargers. To that end, this paper addresses the fast charging station location problem in an urban environment. The optimization problem is formulated as a maximum coverage location problem (MCLP) and existing locations of petrol/fuel stations are considered as candidate locations. Using QGIS software, a geographic information system (GIS) based platform is developed and integrated with a linear-programming relaxation based MCLP algorithm developed in Python. The city of Raleigh, North Carolina with actual geo-spatial data is chosen as a case study. Both census population and highway traffic data are considered as demand metrics to mimic drivers without dedicated chargers and vehicles on highways who need a recharge. A number of evaluations are performed to explore the trade-off between the number of locations and the physical coverage space. Furthermore, comparative analysis show that locating fast chargers in existing petrol stations improve demand coverage by more than $50 \%$ when compared to existing fast charging station locations.
\end{abstract}

Index Terms-electric vehicles, petrol stations, facility location, fast chargers, maximum coverage problem

\section{INTRODUCTION}

Over the next decade, the number of new electric vehicle sales is expected to grow exponentially and substantial investments are needed to build charging infrastructures to enable longer driving distances and provide fast charging service that is comparable with internal combustion engine counterparts [1], [2]. In parallel, the competing downstream oil sector has been in a gradual downsizing and the number of gas/petroleum stations has reduced due to improved fuel efficiencies of combustion engine vehicles and interest for alternative fuel vehicles such as EVs [3]. For instance, the US had 114 thousand petrol stations in 2012, down from 121 thousand in 2002 and, similarly, the number of petrol stations in the UK has declined by one third during the same period [4]. Moreover, petrol station business has been shaped by long-held norms and practices of drivers. Therefore, a natural question arises whether existing petrol stations can be transformed into fast EV charging locations. In this paper, we propose a GISbased facility location problem for EV fast charging stations, examine the existing locations for petrol stations in terms of demand coverage, and compare the coverage with that of already deployed fast charging networks.

Facility location models are optimisation problems that are used to decide on the set of locations to serve customer demand in a network [5]. The overall aim is to optimise one or more quantifiable objectives related to serving customer demand, infrastructure cost, or achieving environmental targets [6]. Charging station infrastructure planning is capital-intensive process that involves one-time decisions and have long-lasting ramifications. In literature, charging station problems are divided into two groups. The first group of studies investigate the optimal locations in a highway network [7]-[9] [10]-[12], while the second group aims to locate stations in an urban environment. For the case of highways, chargers can be located anywhere in the network, hence, these approaches are referred as continuous network models [5]. The facility location problem is solved by solving a flow-capturing refueling problem and the locations of the stations depend on the traffic volume of each origin-destination pair and the all electric range of vehicles.

To locate stations in an urban environment, discrete network models are adopted as the stations can only be located at discrete locations such as existing parking lots or service stations. There are two types of approaches to find the optimal locations of charging facilities. The first one is using classical facility location techniques such as set-covering problems in which the overall aim is to minimise the number of chargers needed in a way that all customers can reach a station within a certain driving distance or time. Set covering problems are further divided into three groups as maximum coverage, $p$-center and p-median problems. In maximum coverage problem, the goal is to maximise the demand coverage for a given number of charging stations. Whereas in p-center problem, the goal is to minimise the maximum driving distance with $\mathrm{P}$ stations. Similarly, in p-median problem median driving range is used as the statistical measure [6], [13]. The second approach is geo-spatial analysis-based multi-criteria decision making 
(MCDM) methods and two recent studies are presented in [14] and [15]. In MCDM approach, each candidate location is evaluated based on different criterion such as cost of the land, availability of parking lots, proximity to green areas, slope of the location. Then a score is assigned to each subcategory and location decisions are made based on ranking the cumulative score of each candidate location.

According to a recent study [16], charging station availability, range anxiety, and refueling duration are three of the major roadblocks for faster EV adoption. Moreover, convenient access to charging facilities has a direct impact on the EV penetration levels. This is particularly because gasoline is considered as a convenience purchase and drivers do not prefer to plan ahead for to find recharging services [13]. Therefore, fast charging stations act as "emergency service" facilities and it is critical to consider behavioral aspects of consumers when locating charging stations. Since the fuel/petroleum retail business is mature and optimised to serve light-duty vehicles, they naturally become good candidates for locating fast EV chargers. In order to address aforementioned issues, we have carried out the following contributions:

- We formulate fast-charging station problem as a maximum coverage facility location problem and identify existing fuel/petroleum stations as the candidate locations. We consider both housing and actual highway traffic data as demand inputs to mimic EV charging demand that could stem from drivers living at multi-dwelling units and without access to night-time charging and pass-by drivers who need to extend their driving range.

- Using QGIS software [17], we develop a GIS-based platform and work with actual maps, candidate station locations, and demand points. We develop maximal coverage algorithm in Python and integrate it with QGIS platform.

- We consider Raleigh, North Carolina as a case study to mimic a mid-side town with nearly half a million population. Station location problem is solved for gradually increasing coverage ranges and compared with existing fast charger locations. Our results indicate that existing petrol stations improve demand coverage by $50 \%$ when compared to already deployed fast chargers.

\section{Problem Formulation}

\section{A. Overview}

In this paper, we formulate the facility location problem as a maximal covering location problem (MCLP) [18] which computes the minimal number and locations of charging stations and ensures that no demand point will be farther than the maximal service distance (e.g. $S \mathrm{~km}$ ) from a station. The rationale behind choosing MCLP over other methods discussed in the previous section is that such facilities will act as an "emergency" service location to beat range anxiety. It is noteworthy that a given geographical region is divided into sub-regions (e.g., zones determined by city councils etc.) and the demand is assumed to occur in centroid of the demand zone. Therefore, if the centroid of a zone is within $S \mathrm{~km}$ away from a charging station that zone is assumed to be covered. Moreover, this approach only considers "coverage" problem and only one charger is sited in a chosen location. "Capacity" problem which addresses the number of physical chargers to be deployed in one station.

\section{B. Problem Formulation}

The MCLP problem is composed of linear and integer values and formulated as follows:

$$
\begin{aligned}
\operatorname{maximise} & z=\sum_{i \in I} a_{i} y_{i} \\
\text { s.t. } & \sum_{j \in N_{i}} x_{i} \geq y_{i} \forall i \in I \\
& \sum_{j \in J} x_{j}=P \\
& x_{j}=(0,1) ; \forall j \in J \\
& y_{i}=(0,1) ; \forall i \in I
\end{aligned}
$$

where $I$ is the set of demand nodes; $J$ is the set of facility sites; $S$ denotes the coverage distance measured in $\mathrm{km}$ and demand points are considered to be uncovered if the distance beyond $S ; d_{i j}$ is the shortest distance from node $i$ to node $j ; a_{i}$ is the population to be served at demand node $i$ and assumed to be at the centre of the node; and $P$ denotes the number of charging stations to be located. Moreover, $x_{j}$ is a binary variable and is set to 1 if a facility is allocated to site $j$ and is set to 0 otherwise. Finally, $N_{i}$ is the set of locations eligible to cover to demand point $i$. Objective function given in (1) aims to maximise the number of charging demand served within the desired driving distance by achieving maximal coverage. Constraint given in (2) shows that $y_{i}$ equals to 1 when one or more stations are established at locations in the set $N_{i}$ (i.e. one or more stations are located within $\mathrm{S} \mathrm{km}$ of demand point $i$ ). The second constraint given in (3) ensures that exactly $P$ number of stations are allocated. Constraints given in (4) and (5) respectively reflect the binary nature of the station siting decisions and demand node coverage.

\section{Solution Method}

The MCLP problem is an NP-hard problem and can be solved effectively using heuristic methods as given in [19] or linear programming (LP) relaxation. Some of the heuristic solutions methods include greedy-add heuristic, greedy-addwith-substitution heuristic, genetic algorithms, and heuristic concentration [19]. LP relaxation results in a nearly optimal result in exchange for computational efficiency. In this paper, Python's PuLP with GLPK package (an LP modeler written in Python) is used to solve the optimisation problem and details about its implementation can be found in [20]. In LP relaxation approach, binary decision variables (e.g., $x_{j}$ and $y_{i}$ ) are transformed into continuous variables between 0 and 1 . This technique transforms the NP-hard optimization problem into a one that is solvable in polynomial time. 


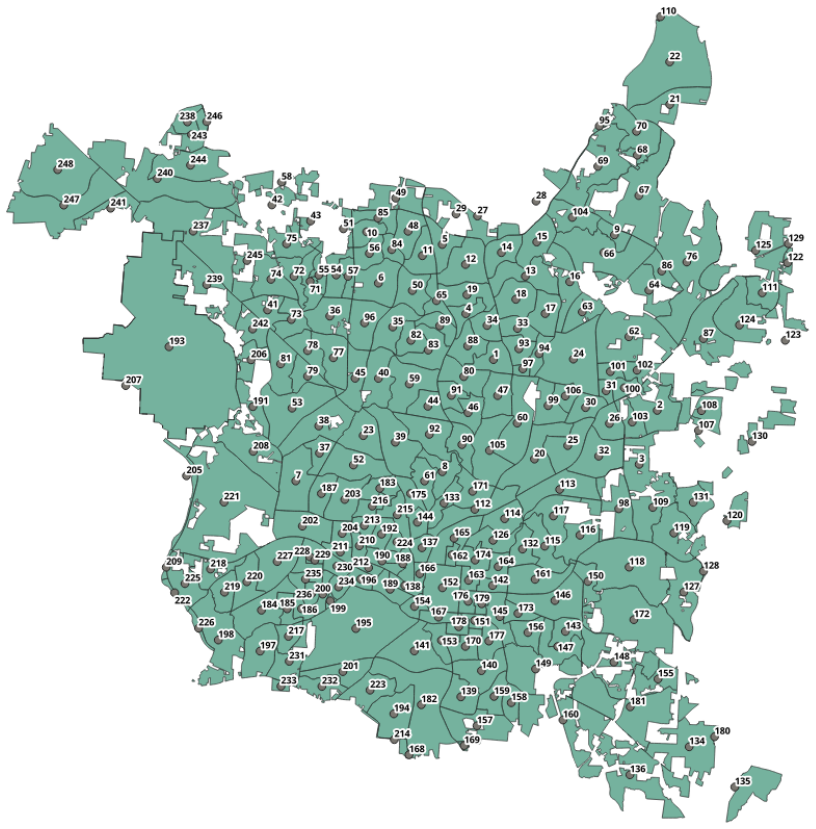

Fig. 1. Raleigh map with census boundaries and Point IDs.

\section{CASE Study}

\section{A. Study Setup}

As a case study, we consider the city of Raleigh, NC by considering the following aspects. Raleigh is one of the fastest growing cities in the United States, its population is nearly half a million, covers a land area of 147.6 square miles, and $78 \%$ of the population drive a car alone to work [21]. Furthermore, $41 \%$ of the population lives in multi-unit housing possibly with limited access to a dedicated charger when EVs become a main mode of transport. The state of North Carolina has introduced plans to push more EVs into the mainstream acceptance [22]. Finally, the city council provides public datasets on traffic, population, and GIS maps which are essential to carry out this study [23].

\section{B. System Inputs}

The city of Raleigh is located in Wake county and the official county website hosts a list of public datasets that are related to locating charging stations. Some datasets are censusblock boundaries of Raleigh, census-data for North Carolina, and the yearly averaged traffic data for North Carolina. It is assumed that the demand points (denoted by set $I$ in constraint (2)) are related to population data and/or traffic data. Hence, the coverage problem aims to locate charging stations to regions with high demand. We use these datasets to get data for block-wise population and traffic. A view of the GIS map with block boundaries is presented in Fig. 1.

Moreover, Fig. 2 shows the highway network along with the centroids of each census blocks. Each bounded demand polygon contains attributes for the population of the specific census block. The demand points are calculated as centroids of the bounded demand polygons. We use a spatial join to

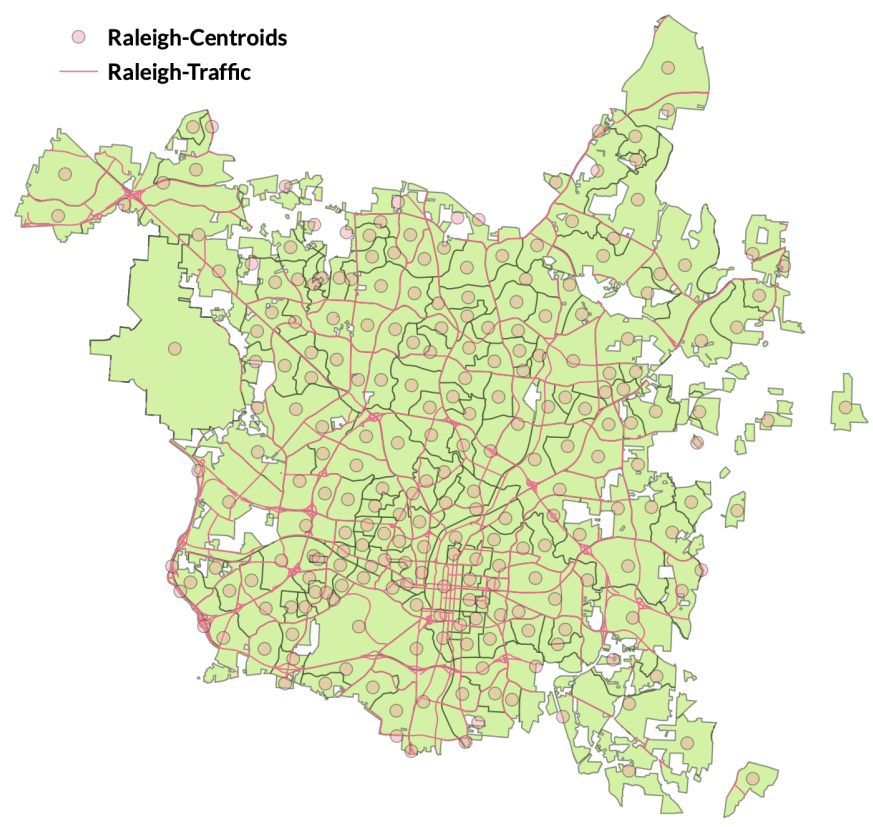

Fig. 2. Demand points and traffic highways in Raleigh, North Carolina

TABLE I

TOP 5 DEMAND POINTS AS PER POPULATION AND TRAFFIC. POINT IDS ARE SHOWN IN FIG. 1.

\begin{tabular}{cccc}
\hline Point-ID & Population Attribute & Point-ID & Traffic Attribute \\
\hline 22 & 8745 & 27 & 97000 \\
76 & 8334 & 64 & 68750 \\
134 & 6970 & 90 & 51800 \\
248 & 6614 & 87 & 50786 \\
21 & 6134 & 226 & 49900 \\
\hline
\end{tabular}

add attributes to the centroids. Note that in GIS operations, a spatial join appends data from one layer's attribute table to another. The traffic data available via the traffic layer is averaged for a demand polygon and added as an attribute to the centroid that contains the highway road. In Table I, a brief description of the attributes available to measure cost for top five demand centroids is presented.

After processing of demand data from Wake county [23], Google Maps is used [24] to obtain the locations of existing fuel stations and fast EV charging stations, and convert the parsed locations to the correct projection for usability in the mapping software. Next, the underlying layer is modified to convert the locations to a polygon type and a coverage radius is added for each facility.

In Fig. 3, the locations of 40 fuel-stations as fetched from Google maps are depicted. After candidate facilities and demand data are imported the modified spatial layers are fed into MCLP algorithm. A Python API for QGIS is used to calculate the distance between regions and to create a matrix of distances for consumption in the algorithm. It is noteworthy that Euclidean distance metric is employed throughout the calculations. As discussed earlier, the MCLP 


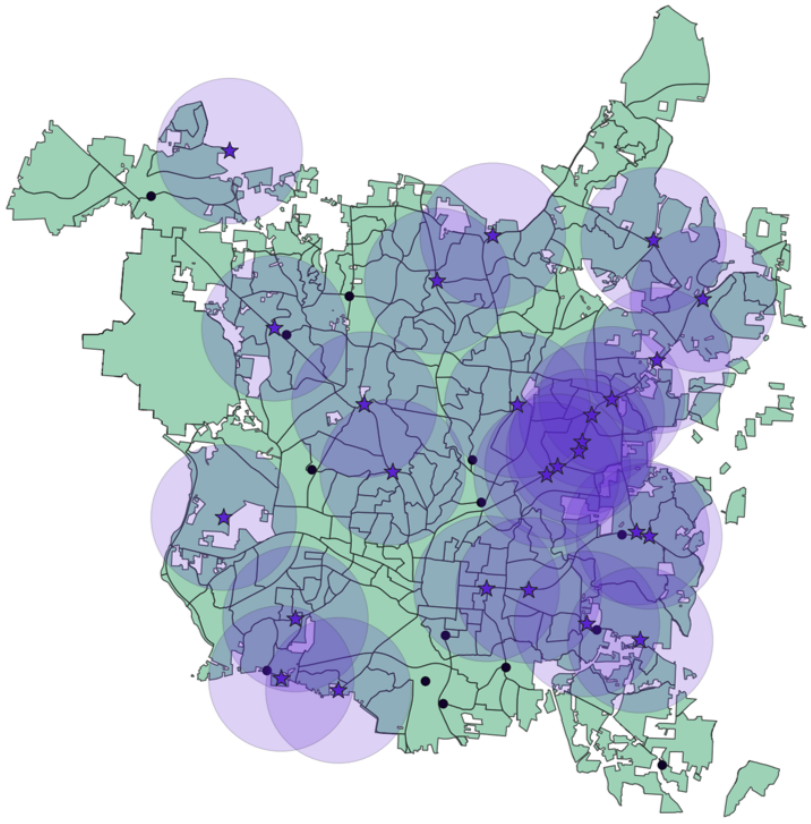

Fig. 3. Optimal Locations of charging stations with a $5 \mathrm{~km}$ diameter.

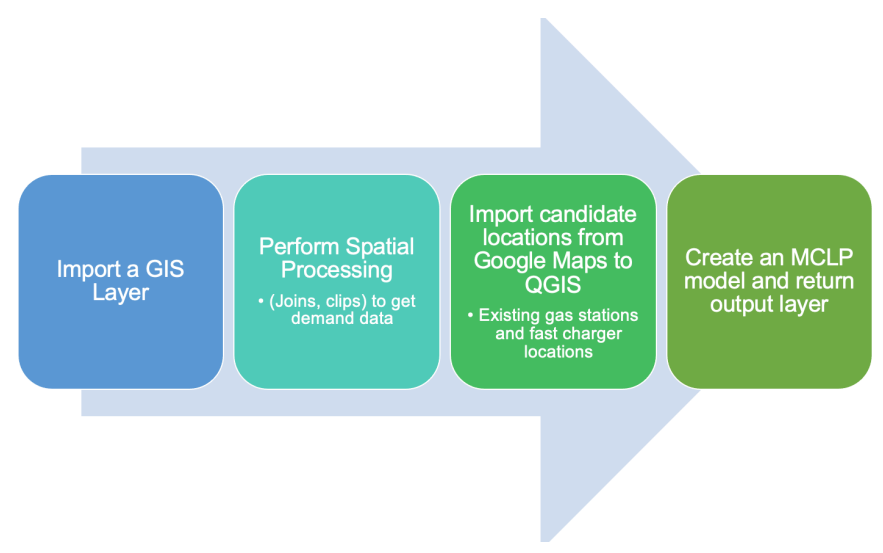

Fig. 4. Methodology for obtaining optimal facility locations.

algorithm takes the number of charging stations, the demand layer, and the facilities layer as inputs. The algorithm locates stations to maximize the demand coverage. The metric that the algorithm uses as "distance" can be modified to include traffic or population. In Fig. 4 a high-level overview of the process to locate charging stations is presented.

\section{Results}

Maximal coverage location problem is solved for a number of case studies to explore the relationship between different system parameters such as demand sources (e.g. population versus traffic), station diameter, the number of stations, and comparison with existing charging station locations. As a first evaluation, MCLP is solved to find the optimal locations of the charging stations from a set of existing fuel/petrol stations to satisfy a $5 \mathrm{~km}$ diameter coverage. In Fig. 3, locations of existing fuel/petrol stations are marked with a black circle and
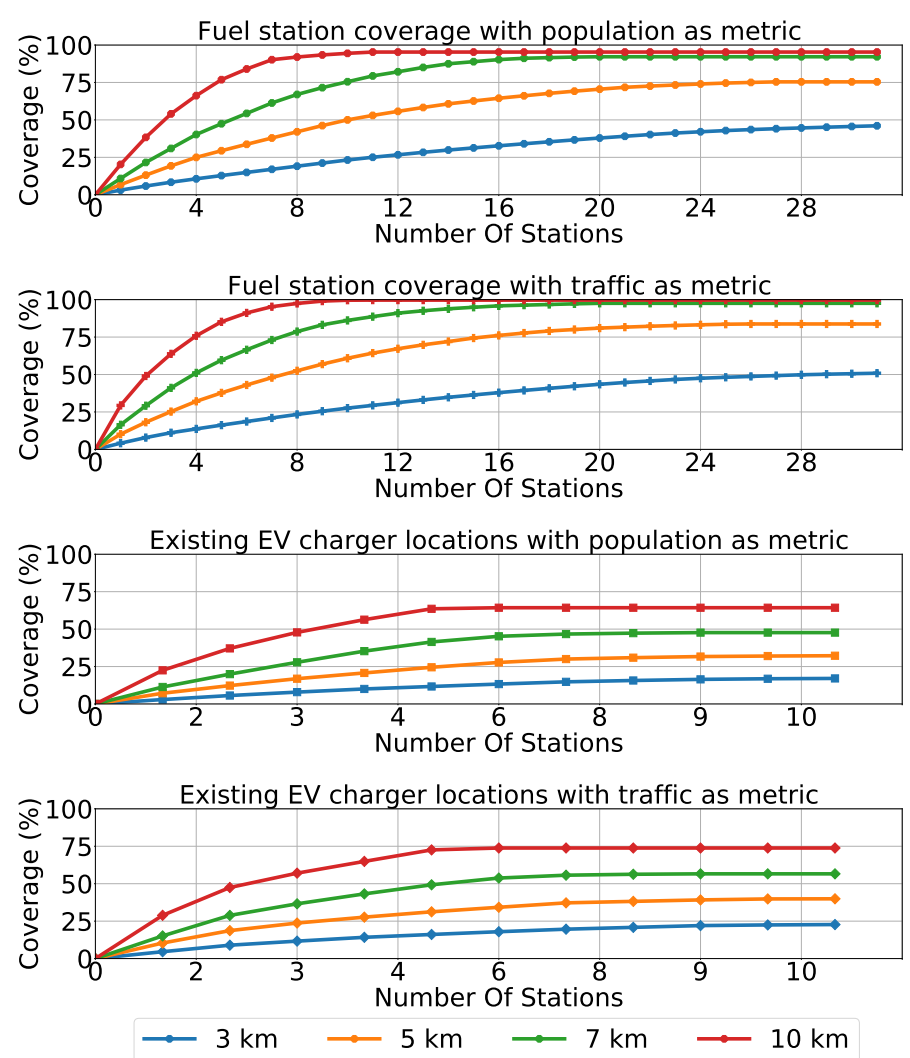

Fig. 5. Optimal facility location using census and road traffic data using existing charging stations and fuel stations as candidate locations.

TABLE II

COMPARISON OF RESULTS BY PERCENTAGE OF COVERED DEMAND.

\begin{tabular}{lcc}
\hline Candidate Location & Diameter & $\begin{array}{c}\text { Max. Possible } \\
\text { Coverage }\end{array}$ \\
\hline $\begin{array}{l}\text { Fuel/Petrol Station } \\
\text { Existing Charging Station }\end{array}$ & \multirow{2}{*}{$\mathrm{km}$} & $\begin{array}{c}46 \% \\
17.3 \%\end{array}$ \\
\hline $\begin{array}{l}\text { Fuel/Petrol Station } \\
\text { Existing Charging Station }\end{array}$ & \multirow{2}{*}{$\mathrm{km}$} & $\begin{array}{c}75.4 \% \\
32.2 \%\end{array}$ \\
\hline $\begin{array}{l}\text { Fuel/Petrol Station } \\
\text { Existing Charging Station }\end{array}$ & \multirow{2}{*}{$\mathrm{km}$} & $\begin{array}{c}92.17 \% \\
47.7 \%\end{array}$ \\
\hline $\begin{array}{l}\text { Fuel/Petrol Station } \\
\text { Existing Charging Station }\end{array}$ & \multirow{2}{*}{$10 \mathrm{~km}$} & $\begin{array}{c}95.3 \% \\
64.2 \%\end{array}$ \\
\hline
\end{tabular}

optimal locations are marked with a star sign. A more detailed analysis is presented in Fig. 5. Proposed algorithm is evaluated for four coverage diameters $(3 \mathrm{~km}, 5 \mathrm{~km}, 7 \mathrm{~km}$, and $10 \mathrm{~km})$ and both census population and road traffic population are considered as demand metric. The location analysis further compared existing fuel and charging stations locations. For the presented urban setting, it is easy to see that locations of the fuel/petrol stations provide a good coverage both for highway traffic demand and population demand. For instance, for the case of $10 \mathrm{~km}$ diameter, more than $90 \%$ of the demand can be served with 7 charging stations when census population is taken as the demand metric, while $90 \%+$ coverage can be achieved with 6 stations when highway traffic is considered as the demand input. 
Fig. 5 further depicts optimal coverage results when existing charging station locations are considered. Note that the existing fast charging station locations are also embedded from Google Maps and contain various fast chargers $(50+\mathrm{kW})$ such as CHAdeMO, EVGo, and Tesla chargers. Since existing chargers are mostly deployed in locations like hotels, shopping malls, and parking spaces, their coverage is considerably lower than petrol stations. Even with $10 \mathrm{~km}$ diameter case, maximum coverage barely reaches to $75 \%$. On the other hand, significantly higher coverage can be achieved with less amount of infrastructure investments.

Main findings are summarized in Table II. Since there are 13 fast charging stations in the region, two candidate locations are compared based on the maximal possible coverage that can be achieved with up to 13 locations. It can be seen that for all coverage ranges, existing fuel/petrol stations provide significantly higher coverage than existing fast charger locations. For a practical diameter target of $7 \mathrm{~km}$, the demand coverage can be nearly doubled if fast chargers are located at existing fuel/petrol stations.

\section{CONCLUSION}

In this paper, optimal location of fast EV charging stations problem was considered. The optimization problem was modeled with a maximum coverage location problem. Unlike existing literature, the location of fuel/petrol stations are considered as candidate sites due to the long-standing capability of fuel/petrol retail business to provide good coverage for light duty vehicle demand. A GIS-based framework was developed to solve the optimization problem using both census population and highway traffic demand data. Actual geo-spatial maps were used and a number of case studies were performed for the city of Raleigh, NC. The results showed that the locations of the existing fuel/petrol stations provide significantly higher coverage than already deployed fast chargers. Such practical results are critical in this early stage of deploying charging infrastructure of the net-zero future.

As a future work, we will investigate the location analysis in a larger metropolitan city and a small one to explore whether the fast charging station coverage can be improved by considering fuel/petrol stations or not. Moreover, optimization problem will be expanded to calculate the optimal number of chargers needed at each location to provide a reasonable quality of service which is often measured by system waiting times or blocking performance.

\section{ACKNOWLEDGMENT}

This publication was made possible by NPRP grant \# NPRP12- from the Qatar National Research Fund (a member of Qatar Foundation). The statements made herein are solely the responsibility of the authors.

\section{REFERENCES}

[1] M. Woodward and et. al., "Electric vehicles setting a course for 2030," Tech. Rep., 2020.

[2] C. Kong, I. S. Bayram, and M. Devetsikiotis, "Revenue optimization frameworks for multi-class pev charging stations," IEEE Access, vol. 3, pp. 2140-2150, 2015.
[3] "Study of the uk petreoleum retail market," Tech. Rep., 2012.

[4] The decline of the petrol station. [Online]. Available: https://eandt.theiet.org/content/articles/2020/03/the-decline-of-thepetrol-station/

[5] S. H. Owen and M. S. Daskin, "Strategic facility location: A review," European journal of operational research, vol. 111, no. 3, pp. 423-447, 1998.

[6] I. S. Bayram and S. Bayhan, "Location analysis of electric vehicle charging stations for maximum capacity and coverage," in 2020 IEEE 14th International Conference on Compatibility, Power Electronics and Power Engineering (CPE-POWERENG), vol. 1. IEEE, 2020, pp. 409414.

[7] C. Kong, R. Jovanovic, I. Bayram, and M. Devetsikiotis, "A hierarchical optimization model for a network of electric vehicle charging stations," Energies, vol. 10, no. 5, p. 675, 2017.

[8] Y. He, K. M. Kockelman, and K. A. Perrine, "Optimal locations of us fast charging stations for long-distance trip completion by battery electric vehicles," Journal of cleaner production, vol. 214, pp. 452-461, 2019.

[9] P. Jochem, E. Szimba, and M. Reuter-Oppermann, "How many fastcharging stations do we need along european highways?" Transportation Research Part D: Transport and Environment, vol. 73, pp. 120-129, 2019.

[10] M. H. Amini, M. P. Moghaddam, and O. Karabasoglu, "Simultaneous allocation of electric vehicles' parking lots and distributed renewable resources in smart power distribution networks," Sustainable cities and society, vol. 28, pp. 332-342, 2017.

[11] A. Y. Lam, Y.-W. Leung, and X. Chu, "Electric vehicle charging station placement: Formulation, complexity, and solutions," IEEE Transactions on Smart Grid, vol. 5, no. 6, pp. 2846-2856, 2014.

[12] T. D. Chen, K. M. Kockelman, and M. Khan, "Locating electric vehicle charging stations: Parking-based assignment method for seattle, washington," Transportation research record, vol. 2385, no. 1, pp. 2836, 2013.

[13] S. Y. He, Y.-H. Kuo, and D. Wu, "Incorporating institutional and spatial factors in the selection of the optimal locations of public electric vehicle charging facilities: A case study of beijing, china," Transportation Research Part C: Emerging Technologies, vol. 67, pp. 131-148, 2016.

[14] D. Guler and T. Yomralioglu, "Suitable location selection for the electric vehicle fast charging station with ahp and fuzzy ahp methods using gis," Annals of GIS, vol. 26, no. 2, pp. 169-189, 2020.

[15] M. Erbaş, M. Kabak, E. Özceylan, and C. Çetinkaya, "Optimal siting of electric vehicle charging stations: A gis-based fuzzy multi-criteria decision analysis," Energy, vol. 163, pp. 1017-1031, 2018.

[16] F. Liao, E. Molin, and B. van Wee, "Consumer preferences for electric vehicles: a literature review," Transport Reviews, vol. 37, no. 3, pp. 252275, 2017.

[17] QGIS a free and open source geographic information system. [Online]. Available: https://www.qgis.org/en/site/

[18] R. Church and C. ReVelle, "The maximal covering location problem," in Papers of the regional science association, vol. 32, no. 1. SpringerVerlag, 1974, pp. 101-118.

[19] M. S. Daskin, Network and discrete location: models, algorithms, and applications. John Wiley \& Sons, 2011.

[20] Optimization with PuLP. [Online]. Available: https://coinor.github.io/pulp/

[21] City of raleigh. [Online]. Available: https://raleighnc.gov/

[22] Road map to more electric vehicles in N.C. [Online]. Available: https://www.ncdot.gov/initiatives-policies/environmental/climatechange/Pages/electric-vehicles.aspx

[23] Wake county GIS maps. [Online]. Available: http://www.wakegov.com/gis/Pages/default.aspx

[24] Google maps places api. [Online]. Available: https://developers.google.com/maps 